\title{
Assistência intensiva às cardiopatias congênitas: Apontamentos ao cuidado de enfermagem neonatal
}

Intensive care for congenital heart diseases: Notes on neonatal nursing care

Cuidados intensivos para cardiopatías congénitas: Notas sobre el cuidado de enfermería neonatal

Laércio Deleon de Melo ORCID: https://orcid.org/0000-0002-8470-7040 Universidade do Estado do Rio de Janeiro, Brasil

E-mail: laerciod128@hotmail.com

Aline Barreto de Araújo

ORCID: https://orcid.org/0000-0002-5092-299X

Centro Universitário Estácio de Juiz de Fora, Brasil

E-mail: barretoaline11@gmail.com

Larissa Gracioso Teixeira

ORCID: https://orcid.org/0000-0003-1079-7431

Centro Universitário Estácio de Juiz de Fora, Brasil

E-mail: larissagracioso@ hotmail.com

Letícia Regina dos Santos

ORCID: https://orcid.org/0000-0003-3245-4333

Centro Universitário Estácio de Juiz de Fora, Brasil

E-mail: leticiatks18@gmail.com

Rosiane de Jesus Pereira

ORCID: https://orcid.org/0000-0002-4381-1730

Faculdade de Ciências Médicas e da Saúde de Juiz de Fora, Brasil

E-mail: heromna@hotmail.com

Maria Tereza Aguiar Castilho Neta Fernandes

ORCID: https://orcid.org/0000-0001-6582-7330

Universidade Federal de Juiz de Fora, Brasil

E-mail: mtneta@hotmail.com

Daniele Lúcia Silva Affonso

ORCID: https://orcid.org/0000-0001-8804-1111

Centro Universitário Estácio de Juiz de Fora, Brasil

E-mail: dani_andrelandia@hotmail.com

Agnes Alvarenga Rosendo

ORCID: https://orcid.org/0000-0002-2287-1670 Centro Universitário Estácio de Juiz de Fora, Brasil

E-mail: agnes.alvarenga@hotmail.com

Paulo Henrique Bezerra Silva

ORCID: https://orcid.org/0000-0002-9057-9102 Centro Universitário Estácio de Juiz de Fora, Brasil

E-mail:ph_1705@yahoo.com.br

Felipe Eduardo Taroco

ORCID: https://orcid.org/0000-0002-8530-5780 Centro Universitário Estácio de Juiz de Fora, Brasil

E-mail: fisiofelipe91@gmail.com

\begin{abstract}
Resumo
Objetivou-se descrever a assistência intensiva de enfermagem cardiovascular a neonatos com cardiopatia congênita. O delineamento adotado foi uma revisão integrativa, cujo período de busca ocorreu entre os meses de agostonovembro/2020 de artigos indexados nos últimos dez anos nas bases de dados. Foram selecionados/inclusos 21 artigos na investigação e elencados os principais cuidados críticos de enfermagem a neonatos portadores de cardiopatias congênitas. Tais cuidados abarcaram a atuação interdisciplinar nos processos de prevenção de doenças, promoção da saúde, diagnóstico precoce de forma clínica, laboratorial e por imagem, bem como nos processos de tratamento clínico, farmacológico ou cirúrgico em prol da reabilitação, cura ou do estabelecimento de um prognóstico favorável à criança. Conclui-se que a atuação do enfermeiro e sua equipe nessa abordagem no contexto intensivo é sistematizada dentro do processo de enfermagem e deve ocorrer de forma interdisciplinar, integrada com os demais profissionais, visando a uma assistência segura, de qualidade, eficaz e humanizada.
\end{abstract}

Palavras-chave: Enfermagem cardiovascular; Cuidados críticos; Cardiopatias congênitas; Neonatologia. 


\begin{abstract}
The objective was to describe the intensive care of cardiovascular nursing to neonates with congenital heart disease. The adopted design was an integrative review, whose search period occurred between the months of AugustNovember/2020 for articles indexed in the last ten years in the databases. 21 articles were selected / included in the investigation and the main critical nursing care for newborns with congenital heart diseases was listed. Such care encompassed interdisciplinary action in the processes of disease prevention, health promotion, early diagnosis in a clinical, laboratory and imaging way, as well as in the processes of clinical, pharmacological or surgical treatment in favor of rehabilitation, cure or the establishment of a favorable prognosis for the child. It is concluded that the role of the nurse and his team in this approach in the intensive context is systematized within the nursing process and must occur in an interdisciplinary way, integrated with the other professionals, aiming at a safe, quality, effective and humanized care.
\end{abstract}

Keywords: Cardiovascular nursing; Critical care; Heart defects, Congenital; Neonatology.

\title{
Resumen
}

El objetivo fue describir los cuidados intensivos de enfermería cardiovascular a neonatos con cardiopatías congénitas. El diseño adoptado fue una revisión integradora, cuyo período de búsqueda se dio entre los meses de agostonoviembre/2020 para los artículos indexados en los últimos diez años en las bases de datos. Se seleccionaron/incluyeron 21 artículos en la investigación y se enumeraron los principales cuidados críticos de enfermería para recién nacidos con cardiopatías congénitas. Dicha atención englobó la actuación interdisciplinar en los procesos de prevención de enfermedades, promoción de la salud, diagnóstico precoz de forma clínica, de laboratorio e imagenológica, así como en los procesos de tratamiento clínico, farmacológico o quirúrgico a favor de la rehabilitación, cura o el establecimiento de un pronóstico favorable para el niño. Se concluye que el rol del enfermero y su equipo en este abordaje en el contexto intensivo está sistematizado dentro del proceso de enfermería y debe ocurrir de manera interdisciplinaria, integrada con los demás profesionales, buscando un cuidado seguro, de calidad, efectivo y humanizado.

Palabras clave: Enfermería cardiovascular; Cuidados críticos; Cardiopatías congénitales; Neonatología.

\section{Introdução}

Segundo Papalia e Feldman (2013), o desenvolvimento cardiovascular é um dos primeiros passos no processo de formação humana, pois nada ocorre ou se forma sem a circulação sanguínea com o fornecimento de oxigênio tecidual. Os ramos vasculares se iniciam na segunda semana de gestação, quando as células mesenquimais se acumulam no saco vitelínico, assim iniciando a formação estrutural do coração.

As paredes da musculatura cardíaca, câmaras, veias, artérias, vasos e valvas se formam até a oitava semana de gestação. Nesse período, ocorre a formação e a multiplicação celular intensa com a diferenciação das estruturas cardiovasculares, sendo este um período de risco em relação à possibilidade de ocorrência de anomalias congênitas, como as cardiovasculares, que podem ocorrer ainda na fase final embrionária (Hockenberry, Wilson \& Rodgers, 2018).

Nesse período inicial de formação e desenvolvimento do Recém-nascido (RN), é de suma importância que este receba acompanhamento e assistência interdisciplinar intensiva, devido a total dependência do neonato, suas fragilidades e possíveis manifestações clínicas decorrentes do processo de adaptação ou ainda à presença de algum atraso no processo de formação, ou presença de malformação e/ou anomalia congênita. Estas são responsáveis pelo óbito precoce ainda na primeira infância, sendo que as Cardiopatias Congênitas (CCs) representam maior morbimortalidade $(40 \%$ das causas, devido à rápida deterioração clínica do neonato e ao alto índice de mortalidade) (Brasil, 2017 a, b; Hockenberry et al., 2018).

A CC é uma má formação na estrutura do coração ou na sua funcionalidade. O coração começa seu desenvolvimento partir do $18^{\circ}$ dia de gestação e, somente a partir da $11^{\circ}$ semana, é possível a identificação da presença de algum tipo de anomalia cardíaca. Essas alterações podem acometer a estrutura muscular, camadas cardíacas, valvas ou vasos sanguíneos (Pinto Júnior et al., 2015; Anwar et al., 2018; Pedra et al., 2019).

O Ministério da Saúde (MS) estima que um a cada cem nascidos vivos no mundo é portador de algum tipo de CC, sendo essa estatística equivalente à brasileira, uma vez que a cada ano ocorrem cerca de 30 mil novos casos no país. Destes, cerca de $20 \%$ possuem mais de um defeito cardíaco e aproximadamente $25 \%$ apresentem defeitos extracardíacos associados. 
Não obstante, do total de RNs com CC cerca de 6\% morrem antes do primeiro ano de vida em decorrência da gravidade da doença (Brasil, 2017 a, b; Saxena et al., 2019).

Diante de tal relevância epidemiológica e do quadro clínico apresentado, bem como das possíveis repercussões durante o crescimento e desenvolvimento da criança, justifica-se a necessidade de tratamento intensivo com abordagem multiprofissional. A assistência à saúde do neonato envolve uma composição por profissionais especializados em diferentes áreas (interdisciplinar), engloba conceitos e métodos e enfrenta intercorrências numa relação de equipe harmônica, com uniformidade de linguagem de forma objetiva e clara em prol do alcance de metas preestabelecidas (Araújo Neto, Silva, Zanin, Andrade \& Moraes, 2016).

A Unidade de Terapia Intensiva (UTI) é uma área hospitalar crítica, indicada para o tratamento de pessoas que necessitam de acompanhamento multiprofissional e interdisciplinar especializado contínuo. Utiliza-se de aporte tecnológico avançado, necessário à monitorização e à terapia, o que contribui para diagnóstico, tratamento e reabilitação clínica e hemodinâmica intensiva. As UTIs podem ser classificadas em: Adulto (UTI-A), Especializada (UTI-E), Neonatal (UTI-N), Pediátrica (UTI-P) e Pediátrica Mista (UTI-Pm) (Brasil, 2010, 2020a; Melo et al., 2020). A neonatologia, como campo de atuação da enfermagem, abrange o cuidado ao neonato desde o momento do seu nascimento até o $28^{\circ}$ dia de vida (Hockenberry et al., 2018).

A interdisciplinaridade, da qual o enfermeiro e sua equipe fazem parte, requer que todos os profissionais envolvidos tenham vínculo, comunicação efetiva e capacitação teórico-técnica para a atuação conjunta, com troca de informações, conhecimentos e experiências trianguladas em prol da resolução clínica das demandas da pessoa cuidada (Brasil, 2017a, b, c).

A partir da relevância apresentada, justifica-se a necessidade de realização desta investigação, sendo corroborada ainda pela Agenda de Prioridades de Pesquisa do Ministério da Saúde (APPMS), que apresenta 14 eixos temáticos de investigação em saúde a serem realizados no Brasil. Entre estes estão relacionados ao objeto investigado os seguintes eixos: 5) Doenças Crônicas Não Transmissíveis (DCNTs); 8) Gestão do trabalho e gestão em saúde e; 14) Saúde materno-infantil (Brasil, 2018), bem como a aprovação do plano nacional de assistência à criança com CC em 2017 (Brasil, 2017b).

A escolha pela temática fundamenta-se ainda na necessidade de enfatizar o assunto no campo da enfermagem e a importância dos cuidados intensivos de enfermagem no contexto das CCs, uma vez que a maioria dos estudos indexados são predominantemente de abordagem médico-cirúrgica. Desse modo, foi elaborada a questão de investigação: quais são os principais cuidados intensivos de enfermagem cardiovascular a neonatos portadores de CC. Objetivou-se, portanto, descrever a assistência intensiva de enfermagem cardiovascular a neonatos com CC.

\section{Metodologia}

Pesquisa qualitativa de delineamento metodológico do tipo revisão integrativa empírica, que visou identificar o estado da arte sobre o objeto investigado com foco em estudos quantitativos e qualitativos sobre o tema pesquisado, bem como na literatura aliada (guilines, diretrizes, legislações, etc.) (Tesmer et al., 2020).

A revisão integrativa deve atender às seis etapas metodológicas, a saber: 1) Identificação do tema e seleção da questão da pesquisa; 2) Estabelecimento dos critérios de inclusão e exclusão; 3) Identificação dos estudos pré-selecionados e selecionados; 4) Categorização dos estudos selecionados; 5) Análise e interpretação dos resultados e 6) Apresentação/síntese do conhecimento científico (Melo, Silva, \& Jeremias, 2021). Essas etapas foram descritas de forma detalhada na Figura 1. 
Figura 1. Etapas da Revisão Integrativa. Juiz de Fora, MG, Brasil, 2021.

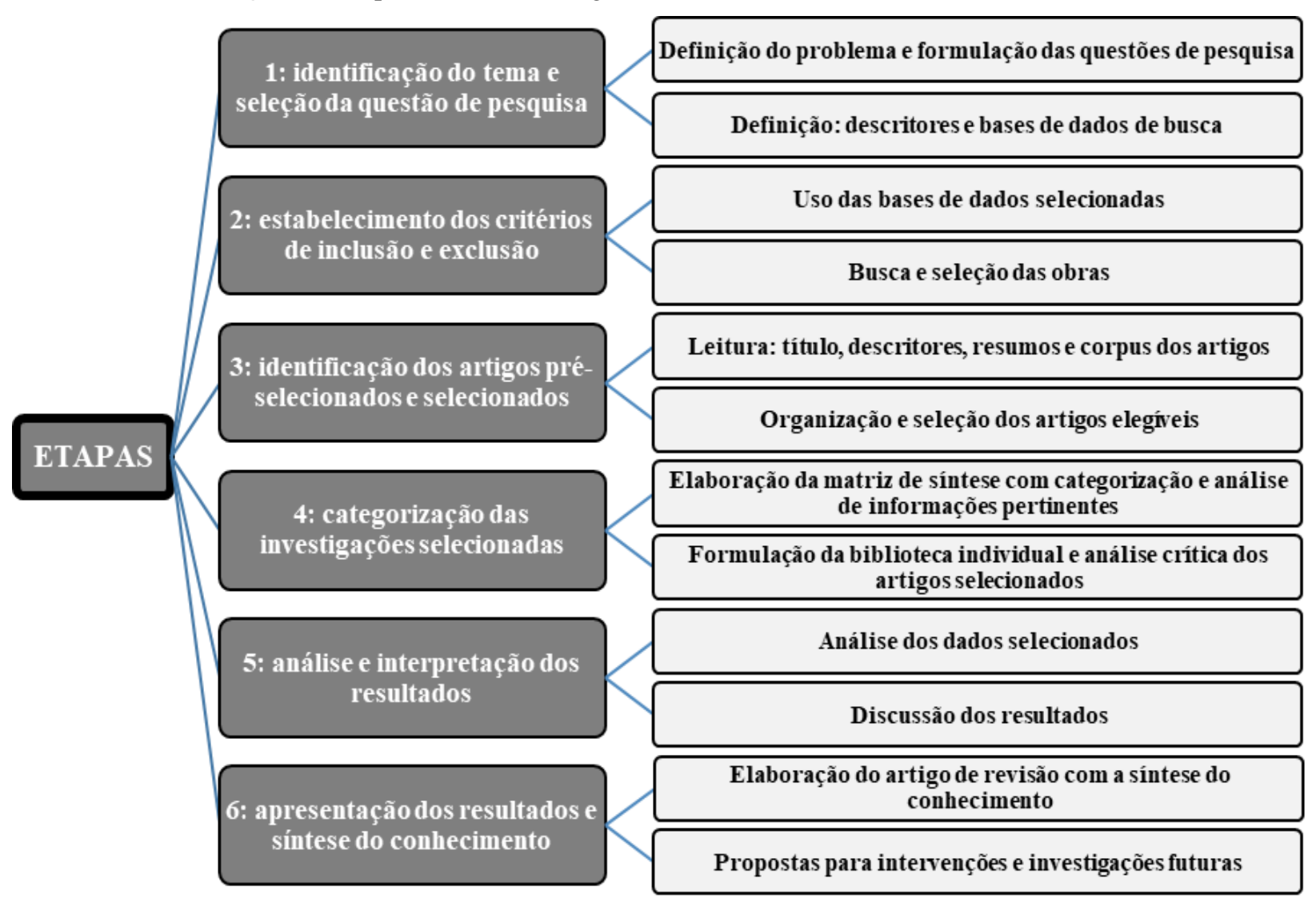

Fonte: Autores, conforme descrição metodológica.

O delineamento de pesquisa do tipo revisão integrativa consiste na análise de artigos indexados em busca da captação de conteúdos e elaboração de uma síntese do conhecimento científico de forma compilada para o estabelecimento de condutas e ações profissionais a partir do conjunto de conhecimentos previamente indexados (inter)nacionalmente (Sousa, Firmino, Marques-Vieira, Severino \& Pestana, 2018; Marques, Melo, Taroco, Duarte \& Lima, 2021).

A etapa de coleta de dados ou busca literária foi realizada por acesso on-line, entre os meses de agosto a novembro de 2020, nas bases de dados: Medical Analyses and Retrieval System Online (Medline); Biblioteca Virtual da Saúde (BVS); Scientific Eletronic Library (Scielo) e Literatura Latino-americana e do Caribe em Ciências da Saúde (Lilacs). Adotou-se o uso combinado de palavras-chave de acordo com o Medical Subject Headings (MeSH) e Descritores em Ciências da Saúde (DeCS), tendo sido adotados os descritores: "Enfermagem Cardiovascular", "Enfermagem”, “Cuidados Críticos”, "Unidades de Terapia Intensiva", "Cardiopatias Congênitas", "Anomalias Congênitas" e "Neonatologia”.

Como recursos de pesquisa foram utilizadas as opções: texto completo disponível na íntegra de acesso livre; limites humanos, neonatologia; idiomas - português, inglês e espanhol, sendo utilizado o operador booleano AND no cruzamento dos descritores. Foram incluídos artigos indexados nos últimos dez anos, visando à captação das evidências científicas atualizadas sobre o objeto investigado. Foram excluídos aqueles artigos que não eram capazes de responder ao objeto de investigação; aqueles cujo delineamento era do tipo relato de caso/experiência e revisão (literária, bibliográfica, narrativa, integrativa ou sistemática). Artigos indexados em mais de uma base foram considerados apenas uma vez.

Os artigos foram analisados primeiramente a partir da leitura individual por cada autor de título, resumo e palavraschave (pré-seleção). Posteriormente, todos os manuscritos que apresentavam potencial para responder ao objeto de 
investigação foram lidos na íntegra por todos os autores para a captação de conteúdos e evidências que justificavam a sua inclusão no estudo (seleção).

\section{Resultados e Discussão}

$\mathrm{Na}$ etapa de pré-seleção, eram 2.023 artigos, dos quais foram excluídos 44 por delineamento do tipo relato de caso/experiência e 23 de revisão; 1.210 não respondiam ao objeto investigado e 725 não estavam disponíveis na íntegra para acesso livre. Desse modo, foram inclusos 21 artigos que se encontram apresentados no Quadro 1, tendo sido a síntese do conhecimento construída e discutida em consonância com a literatura concernente ao tema.

Os artigos foram pré-selecionados e analisados mediante a leitura de título, resumo e descritores no primeiro momento e aqueles que demonstravam potencial para responder à questão de investigação foram lidos na íntegra por todos os autores para a seleção das informações relevantes a serem inclusas na síntese do conhecimento científico. Na construção da síntese do conhecimento acerca dos resultados, predominaram artigos encontrados indexados em revistas especializadas nas áreas de terapia intensiva, urgência e emergência, cardiologia, pediatria, medicina e enfermagem.

Quadro 1: Síntese dos resultados da revisão integrativa. Juiz de Fora, MG, Brasil, 2021.

\begin{tabular}{|c|c|c|c|c|c|}
\hline Periódico & Autores & Título & Objetivos & Métodos & Conclusões \\
\hline \multicolumn{6}{|c|}{ Enfermagem AND “Cuidados Críticos" AND "Cardiopatias Congênitas" AND "Neonatologia" } \\
\hline Pediatrics & $\begin{array}{l}\text { Oster et } \\
\text { al., } 2016 .\end{array}$ & $\begin{array}{l}\text { Lições aprendidas } \\
\text { com exames de } \\
\text { recém-nascidos para } \\
\text { cardiopatias } \\
\text { congênitas críticas }\end{array}$ & $\begin{array}{l}\text { Revisar as práticas } \\
\text { atuais em triagem } \\
\text { neonatal para CCHD e } \\
\text { identificar } \\
\text { oportunidades de } \\
\text { melhoria. }\end{array}$ & $\begin{array}{l}\text { Estudo } \\
\text { qualitativo }\end{array}$ & $\begin{array}{l}\text { A triagem para CCHD com } \\
\text { oximetria de pulso é um importante } \\
\text { programa de saúde pública com o } \\
\text { objetivo de melhorar a vida das } \\
\text { crianças por meio da detecção da } \\
\text { presença de CCHD antes do início } \\
\text { dos sintomas; um benefício } \\
\text { adicional é a identificação de } \\
\text { crianças com hipoxemia devido a } \\
\text { condições secundárias. }\end{array}$ \\
\hline $\begin{array}{l}\text { Cochrane } \\
\text { Database Syst } \\
\text { Rev }\end{array}$ & $\begin{array}{l}\text { Plana et } \\
\text { al., } 2018 .\end{array}$ & $\begin{array}{l}\text { Rastreamento de } \\
\text { oximetria de pulso } \\
\text { para defeitos } \\
\text { cardíacos congênitos } \\
\text { críticos }\end{array}$ & $\begin{array}{l}\text { Determinar a precisão } \\
\text { diagnóstica da } \\
\text { oximetria de pulso } \\
\text { como método de } \\
\text { triagem para detecção } \\
\text { de CCHD em RNs } \\
\text { assintomáticos }\end{array}$ & $\begin{array}{l}\text { Estudo } \\
\text { qualitativo }\end{array}$ & $\begin{array}{l}\text { A oximetria de pulso é um teste } \\
\text { altamente específico e } \\
\text { moderadamente sensível para } \\
\text { detecção de CCHD com taxas de } \\
\text { falso positivo muito baixas. As } \\
\text { evidências atuais apoiam a } \\
\text { introdução de exames de rotina para } \\
\text { CCHD em RNs assintomáticos } \\
\text { antes da alta do berçário de bebês } \\
\text { saudáveis. }\end{array}$ \\
\hline \multicolumn{6}{|c|}{ Enfermagem AND “Cuidados Críticos” AND “Cardiopatias Congênitas” } \\
\hline $\begin{array}{l}\text { The Annals of } \\
\text { thoracic } \\
\text { surgery }\end{array}$ & $\begin{array}{l}\text { Hickey et } \\
\text { al., } 2016 .\end{array}$ & $\begin{array}{l}\text { Impacto da } \\
\text { enfermagem em } \\
\text { cuidados intensivos } \\
\text { nos resultados de } \\
\text { pacientes pediátricos }\end{array}$ & $\begin{array}{l}\text { Descrever o efeito da } \\
\text { experiência e educação } \\
\text { de enfermagem em } \\
\text { operações cardíacas } \\
\text { pediátricas pós- } \\
\text { operatórias. }\end{array}$ & $\begin{array}{l}\text { Estudo } \\
\text { retrospectivo } \\
\text { qualitativo }\end{array}$ & $\begin{array}{l}\text { Esses resultados fornecem dados } \\
\text { para líderes de hospitais pediátricos } \\
\text { e reforçam a importância de } \\
\text { estratégias de mentoria em toda a } \\
\text { organização para novos enfermeiros } \\
\text { e estratégias de retenção para } \\
\text { enfermeiros experientes. }\end{array}$ \\
\hline $\begin{array}{r}\text { Escola Anna } \\
\text { Nery }\end{array}$ & $\begin{array}{l}\text { Melo et al., } \\
2012 \text {. }\end{array}$ & $\begin{array}{l}\text { O ser-enfermeiro em } \\
\text { face do cuidado à } \\
\text { criança no pós- } \\
\text { operatório imediato } \\
\text { de cirurgia cardíaca. }\end{array}$ & $\begin{array}{l}\text { Compreender o } \\
\text { cuidado à criança } \\
\text { durante o pós- } \\
\text { operatório imediato de } \\
\text { cirurgia cardíaca a } \\
\text { partir da percepção do } \\
\text { ser-enfermeiro }\end{array}$ & $\begin{array}{l}\text { Estudo } \\
\text { qualitativo } \\
\text { fenomenológico }\end{array}$ & $\begin{array}{l}\text { O cuidado à criança é construído em } \\
\text { meio a uma dinâmica constitutiva } \\
\text { entre tecnologias duras e } \\
\text { subjetividade, na qual haverá } \\
\text { momentos em que uma será } \\
\text { valorizada em detrimento da outra. }\end{array}$ \\
\hline
\end{tabular}




\begin{tabular}{|c|c|c|c|c|c|}
\hline \multicolumn{6}{|c|}{ “Cuidados Críticos” AND “Cardiopatias Congênitas" } \\
\hline Cureus & $\begin{array}{l}\text { Khokhar, } \\
\text { Gowa, } \\
\text { Bangash, } \\
\text { \& Tahir, } \\
2019 .\end{array}$ & $\begin{array}{l}\text { O espectro de } \\
\text { procedimentos } \\
\text { cardíacos pediátricos } \\
\text { e seus resultados: um } \\
\text { relatório de seis } \\
\text { meses da maior } \\
\text { instalação cardíaca } \\
\text { em Sindh, Paquistão }\end{array}$ & $\begin{array}{l}\text { Analisar o espectro de } \\
\text { procedimentos } \\
\text { cirúrgicos cardíacos } \\
\text { pediátricos realizados } \\
\text { para o tratamento de } \\
\text { doença cardíaca } \\
\text { congênita e seus } \\
\text { resultados em uma } \\
\text { UTI-P cardíaca no } \\
\text { Paquistão. }\end{array}$ & $\begin{array}{l}\text { Estudo } \\
\text { quantitativo }\end{array}$ & $\begin{array}{l}\text { Há uma carga muito alta de } \\
\text { pacientes nas UTIs-P cardíacas em } \\
\text { países asiáticos de renda baixa a } \\
\text { média. Apesar da falta de recursos, } \\
\text { o atendimento de alta qualidade } \\
\text { prestado pelos especialistas em } \\
\text { cuidados intensivos em cardiologia } \\
\text { pediátrica nessas UTI-P tem } \\
\text { garantido resultados favoráveis e } \\
\text { uma taxa de mortalidade tão baixa } \\
\text { quanto à de qualquer um dos países } \\
\text { desenvolvidos. }\end{array}$ \\
\hline PLoS One & $\begin{array}{l}\text { Mebius et } \\
\text { al., } 2020 .\end{array}$ & $\begin{array}{l}\text { Início de lesão } \\
\text { cerebral em bebês } \\
\text { com Cardiopatia } \\
\text { Congênita } \\
\text { Diagnosticada (DAC) } \\
\text { no pré-natal }\end{array}$ & $\begin{array}{l}\text { Avaliar a associação } \\
\text { entre os padrões de } \\
\text { fluxo Doppler pré- } \\
\text { natal, a oxigenação } \\
\text { cerebral pós-natal e o } \\
\text { resultado neurológico } \\
\text { em curto prazo. }\end{array}$ & $\begin{array}{l}\text { Estudo } \\
\text { qualitativo }\end{array}$ & $\begin{array}{l}\text { Em bebês com diagnóstico pré-natal } \\
\text { de DAC, o período pré-natal pode } \\
\text { desempenhar um papel importante } \\
\text { no resultado do } \\
\text { desenvolvimento. Pesquisas } \\
\text { adicionais são necessárias para } \\
\text { esclarecer a relação entre a } \\
\text { oxigenação cerebral pré-operatória, } \\
\text { intraoperatória e pós-operatória e o } \\
\text { resultado do desenvolvimento em } \\
\text { bebês com diagnóstico pré-natal de } \\
\text { DAC }\end{array}$ \\
\hline Transl Pediatr & $\begin{array}{l}\text { Khalil et } \\
\text { al., } 2019 .\end{array}$ & $\begin{array}{l}\text { Terapia aguda de } \\
\text { recém-nascidos com } \\
\text { doença cardíaca } \\
\text { congênita crítica }\end{array}$ & $\begin{array}{l}\text { Identificar as mais } \\
\text { adequadas terapias } \\
\text { cirúrgicas ou } \\
\text { procedimentos que } \\
\text { possam proporcionar } \\
\text { melhorias ao recém- } \\
\text { nascido cardiopata. }\end{array}$ & $\begin{array}{l}\text { Estudo } \\
\text { qualitativo }\end{array}$ & $\begin{array}{l}\text { A terapia aguda do RN com doença } \\
\text { cardíaca congênita crítica requer, no } \\
\text { entanto, uma equipe cardíaca bem } \\
\text { preparada e um estoque de cateter } \\
\text { suficientemente equipado. }\end{array}$ \\
\hline $\begin{array}{l}\text { Braz J } \\
\text { Cardiovasc } \\
\text { Surg }\end{array}$ & $\begin{array}{l}\text { Rocha et } \\
\text { al., } 2018\end{array}$ & $\begin{array}{l}\text { Fatores de risco para } \\
\text { mortalidade em } \\
\text { crianças com } \\
\text { cardiopatia congênita } \\
\text { internadas em um } \\
\text { centro terciário } \\
\text { brasileiro }\end{array}$ & $\begin{array}{l}\text { Investigar a incidência } \\
\text { do diagnóstico pós- } \\
\text { natal de CC e os fatores } \\
\text { preditivos de } \\
\text { mortalidade hospitalar. }\end{array}$ & $\begin{array}{l}\text { Estudo de } \\
\text { coorte } \\
\text { retrospectivo }\end{array}$ & $\begin{array}{l}\text { A incidência pós-natal de DAC foi } \\
\text { de } 5,3 \% \text {. Baixo } 1 \text { pontuação min de } \\
\text { Apgar, de baixo peso de } \\
\text { nascimento, e a complexidade DCC } \\
\text { foram os fatores independentes que } \\
\text { afetaram o resultado do hospital. }\end{array}$ \\
\hline $\begin{array}{l}\text { JACC Basic } \\
\text { Transl Sci }\end{array}$ & $\begin{array}{l}\text { Anwar et } \\
\text { al., } 2018 \text {. }\end{array}$ & $\begin{array}{l}\text { Impressão 3D é uma } \\
\text { tecnologia } \\
\text { transformadora em } \\
\text { doenças cardíacas } \\
\text { congênitas }\end{array}$ & $\begin{array}{l}\text { Descrever o papel } \\
\text { dessa tecnologia de } \\
\text { impressão 3D na } \\
\text { comunicação com } \\
\text { equipes } \\
\text { multidisciplinares, } \\
\text { pacientes e cuidadores. }\end{array}$ & $\begin{array}{l}\text { Estudo } \\
\text { qualitativo }\end{array}$ & $\begin{array}{l}\text { Os modelos 3D podem levar a } \\
\text { avanços médicos ao permitir a } \\
\text { ciência básica, as investigações } \\
\text { translacionais e clínicas. Mais dados } \\
\text { são necessários para quantificar } \\
\text { esses benefícios potenciais da } \\
\text { impressão 3D, e a experiência } \\
\text { inicial é promissora. }\end{array}$ \\
\hline $\begin{array}{l}\text { Rev Bras Cir } \\
\text { Cardiovasc }\end{array}$ & $\begin{array}{l}\text { Pinto } \\
\text { Júnior et } \\
\text { al., } 2015 .\end{array}$ & $\begin{array}{l}\text { Epidemiologia da } \\
\text { cardiopatia congênita } \\
\text { no Brasil }\end{array}$ & $\begin{array}{l}\text { Estimar a } \\
\text { subnotificação da } \\
\text { prevalência de CC no } \\
\text { Brasil e seus subtipos. }\end{array}$ & $\begin{array}{l}\text { Estudo } \\
\text { qualitativo }\end{array}$ & $\begin{array}{l}\text { No Brasil, há subnotificação na } \\
\text { prevalência de CC, sinalizando a } \\
\text { necessidade de ajustes na } \\
\text { metodologia de registro. }\end{array}$ \\
\hline \multicolumn{6}{|c|}{ Unidades de Terapia Intensiva" AND "Cardiopatias Congênitas } \\
\hline $\begin{array}{l}\text { American } \\
\text { Journal of } \\
\text { Perinatology }\end{array}$ & $\begin{array}{l}\text { Martin, } \\
\text { Cross, } \\
\text { Hom \& } \\
\text { Klugman, } \\
2019 .\end{array}$ & $\begin{array}{l}\text { Atualizações em } \\
\text { doenças cardíacas } \\
\text { congênitas: os } \\
\text { resultados podem } \\
\text { melhorar? }\end{array}$ & $\begin{array}{l}\text { Discutir o impacto da } \\
\text { triagem de oximetria de } \\
\text { pulso para DAC crítica, } \\
\text { descrever as } \\
\text { contribuições da } \\
\text { imagem cardíaca } \\
\text { avançada em neonatos } \\
\text { com DAC e mostrar a } \\
\text { crescente importância } \\
\text { da melhoria da } \\
\text { qualidade e dos } \\
\text { programas de }\end{array}$ & $\begin{array}{l}\text { Estudo } \\
\text { qualitativo }\end{array}$ & $\begin{array}{l}\text { Por meio da evolução de esforços } \\
\text { como a Cardiac Networks United, a } \\
\text { melhoria da qualidade multicêntrica } \\
\text { e o compartilhamento de dados } \\
\text { continuarão a promover o cuidado } \\
\text { de crianças com DCC crítica. }\end{array}$ \\
\hline
\end{tabular}




\begin{tabular}{|c|c|c|c|c|c|}
\hline & & & $\begin{array}{l}\text { segurança na UTI } \\
\text { cardíaca }\end{array}$ & & \\
\hline $\begin{array}{l}\text { The Annals of } \\
\text { thoracic } \\
\text { surgery }\end{array}$ & $\begin{array}{l}\text { Bell et al., } \\
2019 .\end{array}$ & $\begin{array}{l}\text { Lesão neurológica } \\
\text { aguda em crianças } \\
\text { internadas em } \\
\text { unidade de terapia } \\
\text { intensiva cardíaca }\end{array}$ & $\begin{array}{l}\text { Nossa hipótese era de } \\
\text { que as lesões } \\
\text { neurológicas agudas } \\
\text { contribuem para a } \\
\text { mortalidade dessas } \\
\text { crianças e são uma } \\
\text { importante causa de } \\
\text { morte. }\end{array}$ & $\begin{array}{l}\text { Estudo } \\
\text { retrospectivo } \\
\text { qualitativo }\end{array}$ & $\begin{array}{l}\text { Os ANEs estão associados a um } \\
\text { aumento na mortalidade e o uso de } \\
\text { ECMO e a parada cardíaca foram } \\
\text { independentemente associados aos } \\
\text { ANEs. Esta população pode se } \\
\text { beneficiar de uma vigilância } \\
\text { aprimorada para identificar e tratar } \\
\text { esses eventos e prevenir resultados } \\
\text { neurológicos desfavoráveis. }\end{array}$ \\
\hline Esc. Anna Nery & $\begin{array}{l}\text { Silva, } \\
\text { Stipp, } \\
\text { Pereira, } \\
\text { Paes \& } \\
\text { Knupp, } \\
2018 .\end{array}$ & $\begin{array}{l}\text { Variáveis clínicas e } \\
\text { laboratoriais } \\
\text { associadas ao } \\
\text { desfecho mortalidade } \\
\text { no pós-operatório de } \\
\text { cirurgia cardíaca } \\
\text { pediátrica }\end{array}$ & $\begin{array}{l}\text { Identificar as variáveis } \\
\text { clínicas e laboratoriais } \\
\text { associadas ao desfecho } \\
\text { mortalidade no pós- } \\
\text { operatório de cirurgia } \\
\text { cardíaca pediátrica. }\end{array}$ & $\begin{array}{l}\text { Estudo } \\
\text { descritivo }\end{array}$ & $\begin{array}{l}\text { A identificação dessas variáveis } \\
\text { configura fator preponderante para } \\
\text { o controle das principais } \\
\text { complicações decorrentes do pós- } \\
\text { operatório, possibilitando o } \\
\text { reconhecimento de alterações } \\
\text { clínicas sutis, exigindo atenção e } \\
\text { intervenção imediata, com } \\
\text { consequente redução da } \\
\text { mortalidade. }\end{array}$ \\
\hline \multicolumn{6}{|c|}{ "Anomalias Congênitas" AND "Neonatologia" } \\
\hline $\begin{array}{l}\text { Revista } \\
\text { Bioética }\end{array}$ & $\begin{array}{l}\text { Gozzola, } \\
\text { Leite \& } \\
\text { Gonçalves, } \\
2020 .\end{array}$ & $\begin{array}{l}\text { Comunicando más } \\
\text { notícias sobre } \\
\text { malformações } \\
\text { congênitas: reflexões } \\
\text { bioéticas e jurídicas }\end{array}$ & $\begin{array}{l}\text { Discutir os aspectos } \\
\text { relacionados à } \\
\text { adequada comunicação } \\
\text { na relação médico- } \\
\text { paciente, fundamental } \\
\text { ao exercício da } \\
\text { autonomia e ao dever } \\
\text { de informar, cuja } \\
\text { violação é passível de } \\
\text { responsabilização nas } \\
\text { esferas cível e ético- } \\
\text { profissional. }\end{array}$ & $\begin{array}{l}\text { Estudo } \\
\text { qualitativo }\end{array}$ & $\begin{array}{l}\text { A comunicação de más notícias não } \\
\text { é tarefa simples, especialmente nos } \\
\text { campos da medicina fetal e da } \\
\text { neonatologia, que cuidam de fases } \\
\text { da vida da mulher e do casal } \\
\text { naturalmente permeadas de } \\
\text { simbolismo, grandes expectativas e } \\
\text { ansiedade: a gravidez e o } \\
\text { nascimento de um filho. }\end{array}$ \\
\hline $\begin{array}{l}\text { Arq Bras } \\
\text { Cardiol }\end{array}$ & $\begin{array}{l}\text { Pedra et } \\
\text { al., } 2019 .\end{array}$ & $\begin{array}{l}\text { Diretriz Brasileira de } \\
\text { Cardiologia Fetal }\end{array}$ & $\begin{array}{l}\text { Diante deste } \\
\text { documento } \\
\text { extremamente } \\
\text { completo e de grande } \\
\text { utilidade, aceitamos o } \\
\text { desafio de reunir } \\
\text { profissionais que se } \\
\text { dedicam à Cardiologia } \\
\text { Fetal das diferentes } \\
\text { regiões do Brasil para } \\
\text { estabelecermos, juntos, } \\
\text { algumas diretrizes } \\
\text { adaptadas à nossa } \\
\text { realidade, levando em } \\
\text { consideração também o } \\
\text { conhecimento gerado } \\
\text { no país. }\end{array}$ & $\begin{array}{l}\text { Estudo } \\
\text { qualitativo }\end{array}$ & $\begin{array}{l}\text { A maioria das CCs não é letal e o } \\
\text { tratamento clássico paliativo no } \\
\text { período neonatal também é uma } \\
\text { opção na maioria das situações. } \\
\text { Entretanto, para algumas anomalias } \\
\text { em que a mudança da história } \\
\text { natural para melhor é possível, ou } \\
\text { para aquelas com um prognóstico } \\
\text { extremamente sombrio, a } \\
\text { intervenção fetal pode ser uma } \\
\text { opção terapêutica. }\end{array}$ \\
\hline $\begin{array}{l}\text { Revista } \\
\text { Brasileira de } \\
\text { Ciências da } \\
\text { Saúde }\end{array}$ & $\begin{array}{l}\text { Aragão et } \\
\text { al., } 2013 .\end{array}$ & $\begin{array}{l}\text { O Perfil } \\
\text { Epidemiológico dos } \\
\text { Pacientes com } \\
\text { Cardiopatia } \\
\text { Congênita } \\
\text { Submetidos à } \\
\text { Cirurgia no Hospital } \\
\text { do Coração }\end{array}$ & $\begin{array}{l}\text { Descrever o perfil } \\
\text { epidemiológico dos } \\
\text { pacientes com CC } \\
\text { submetidos à cirurgia } \\
\text { no hospital do coração. }\end{array}$ & $\begin{array}{l}\text { Estudo } \\
\text { transversal } \\
\text { descritivo } \\
\text { exploratório }\end{array}$ & $\begin{array}{l}\text { Os achados apontaram que o perfil } \\
\text { dos pacientes com CC foi de } \\
\text { lactentes, pré-escolares e escolares, } \\
\text { sem predomínio de gênero. Ocorreu } \\
\text { predomínio das CCs acianóticas, } \\
\text { sendo que a maioria do número de } \\
\text { óbitos estava entre as CCs } \\
\text { cianóticas. }\end{array}$ \\
\hline $\begin{array}{l}\text { Int. J. } \\
\text { Cardiovasc. } \\
\text { Sci. }\end{array}$ & $\begin{array}{l}\text { Jesus et al., } \\
2018 \text {. }\end{array}$ & $\begin{array}{l}\text { Fila de Espera para } \\
\text { Tratamento de } \\
\text { Pacientes com } \\
\text { Cardiopatia } \\
\text { Congênita: retrato de } \\
\text { um Centro de }\end{array}$ & $\begin{array}{l}\text { Analisar o tempo de } \\
\text { espera para realização } \\
\text { de tratamento eletivo } \\
\text { cirúrgico e/ou } \\
\text { intervencionista de } \\
\text { crianças portadoras de }\end{array}$ & $\begin{array}{l}\text { Estudo } \\
\text { transversal }\end{array}$ & $\begin{array}{l}\text { Grande parte das crianças que } \\
\text { aguardam por procedimento } \\
\text { cardíaco é procedente de fora da } \\
\text { região metropolitana e tem } \\
\text { malformações potencialmente } \\
\text { tratáveis por cateterismo. É }\end{array}$ \\
\hline
\end{tabular}




\begin{tabular}{|c|c|c|c|c|c|}
\hline & & $\begin{array}{l}\text { Referência } \\
\text { Amazônico. }\end{array}$ & $\begin{array}{l}\text { CC em um centro de } \\
\text { referência cardiológico } \\
\text { e fazer considerações } \\
\text { sobre as CCs e suas } \\
\text { formas de tratamento } \\
\text { na referida instituição. }\end{array}$ & & $\begin{array}{l}\text { necessário aumentar a capacidade } \\
\text { operacional do único centro de } \\
\text { referência público do estado, além } \\
\text { de descentralizar o atendimento em } \\
\text { alta complexidade cardiológica da } \\
\text { região metropolitana. }\end{array}$ \\
\hline \multicolumn{6}{|c|}{ Enfermagem AND "Cardiopatias Congênitas" } \\
\hline $\begin{array}{l}\text { Revista } \\
\text { Interdisciplinar }\end{array}$ & $\begin{array}{l}\text { Silva, } \\
\text { Aguiar, } \\
\text { Cunha \& } \\
\text { Rodrigues, } \\
2014 .\end{array}$ & $\begin{array}{l}\text { Caracterização do } \\
\text { diagnóstico e } \\
\text { tratamento } \\
\text { farmacológico das } \\
\text { cardiopatias } \\
\text { congênitas neonatais: } \\
\text { acianogênica e } \\
\text { cianogênica }\end{array}$ & $\begin{array}{l}\text { Identificar o sexo, o } \\
\text { peso e o tipo de CC } \\
\text { quanto à classificação } \\
\text { em acianogênica ou } \\
\text { cianogênica; levantar } \\
\text { os fármacos, os } \\
\text { métodos do } \\
\text { diagnóstico, exames e } \\
\text { complementares } \\
\text { durante internação do } \\
\text { cardiopata. }\end{array}$ & $\begin{array}{l}\text { Estudo } \\
\text { quantitativo }\end{array}$ & $\begin{array}{l}\text { Evidenciou-se a predominância de } \\
\text { um variado tipo de exames e } \\
\text { tratamento farmacológico, essas } \\
\text { práticas requerem do enfermeiro um } \\
\text { planejamento adequado acerca dos } \\
\text { horários, da manipulação e da } \\
\text { prevenção de possíveis } \\
\text { complicações ou sequelas. }\end{array}$ \\
\hline $\begin{array}{l}\text { Acta Paulista } \\
\text { de } \\
\text { Enfermagem }\end{array}$ & $\begin{array}{l}\text { Silva, } \\
\text { Pereira, } \\
\text { Figueiredo, } \\
\text { Guimarães } \\
\& \\
\text { Cavalcanti, } \\
2015 .\end{array}$ & $\begin{array}{l}\text { Diagnósticos de } \\
\text { Enfermagem em } \\
\text { crianças com } \\
\text { cardiopatias } \\
\text { congênitas: } \\
\text { mapeamento cruzado }\end{array}$ & $\begin{array}{l}\text { Identificar } \\
\text { Diagnósticos de } \\
\text { Enfermagem da } \\
\text { NANDA Internacional } \\
\text { a partir dos termos } \\
\text { encontrados nos } \\
\text { registros de } \\
\text { Enfermagem de } \\
\text { crianças com CC } \\
\text { hospitalizadas e } \\
\text { verificar associação } \\
\text { entre estes termos e os } \\
\text { Diagnósticos de } \\
\text { Enfermagem } \\
\text { mapeados. }\end{array}$ & $\begin{array}{l}\text { Estudo } \\
\text { observacional, } \\
\text { transversal. }\end{array}$ & $\begin{array}{l}\text { Observou-se que os termos } \\
\text { registrados em prontuários de } \\
\text { crianças com CC permitiram a } \\
\text { identificação dos Diagnósticos de } \\
\text { Enfermagem da NANDA } \\
\text { Internacional, além da verificação } \\
\text { das associações. }\end{array}$ \\
\hline $\begin{array}{l}\text { Revista de } \\
\text { Pesquisa } \\
\text { Cuidado é } \\
\text { Fundamental } \\
\text { Online }\end{array}$ & $\begin{array}{l}\text { Urakawa } \\
\& \\
\text { Kobayashi } \\
(2012)\end{array}$ & $\begin{array}{l}\text { Identificação do } \\
\text { perfil e diagnósticos } \\
\text { de enfermagem do } \\
\text { neonato com } \\
\text { cardiopatia congênita }\end{array}$ & $\begin{array}{l}\text { Caracterizar o perfil e } \\
\text { os diagnósticos de } \\
\text { enfermagem do } \\
\text { neonato com CC. }\end{array}$ & $\begin{array}{l}\text { Estudo } \\
\text { descritivo, } \\
\text { exploratório e } \\
\text { retrospectivo. }\end{array}$ & $\begin{array}{l}\text { Conhecer as características do } \\
\text { neonato com CC favorece a } \\
\text { prestação da assistência de } \\
\text { enfermagem direcionada pelos } \\
\text { diagnósticos de enfermagem }\end{array}$ \\
\hline $\begin{array}{l}\text { Texto \& } \\
\text { Contexto } \\
\text { Enfermagem }\end{array}$ & $\begin{array}{l}\text { Magalhães, } \\
\text { Chaves \& } \\
\text { Queiroz, } \\
2019 .\end{array}$ & $\begin{array}{l}\text { Design instrucional } \\
\text { para o cuidado de } \\
\text { enfermagem aos } \\
\text { neonatos com } \\
\text { cardiopatias } \\
\text { congênitas }\end{array}$ & $\begin{array}{l}\text { Desenvolver e validar } \\
\text { design instrucional } \\
\text { para o cuidado clínico } \\
\text { de Enfermagem aos } \\
\text { neonatos com CC em } \\
\text { maternidades, por meio } \\
\text { de educação a } \\
\text { distância. }\end{array}$ & $\begin{array}{l}\text { Estudo } \\
\text { quantitativo }\end{array}$ & $\begin{array}{l}\text { O design instrucional foi validado } \\
\text { pela congruência da opinião dos } \\
\text { juízes ( } 85 \% \text { ) e a estratégia de } \\
\text { validação incorporou diferentes } \\
\text { saberes, qualificando a tecnologia e } \\
\text { apontando a importância da } \\
\text { temática na capacitação de } \\
\text { enfermeiros, com recursos da } \\
\text { Educação a Distância. }\end{array}$ \\
\hline
\end{tabular}

Nota: Autores, conforme busca nas bases de dados. (25/10/2020).

As malformações anatômicas do coração são consideradas críticas no contexto das CCs, que se subdividem em: cianóticas - apresentam maior risco para o neonatal, acarretando menor fluxo sanguíneo pulmonar no Canal Arterial (CA) dependente, no qual ocorre uma cianose típica em pele e mucosas, indicando hipoxemia generalizada. São elas: Tetralogia de Fallot (TF), atresia tricúspide, atresia pulmonar, estenose pulmonar, coração univentricular com estenose pulmonar e estenose pulmonar crítica e Transposição das Grandes Artérias (TGA), as quais tendem a ser fatais nos primeiros dias de vida se não houver intervenção nas primeiras horas de vida; acianóticas - com fluxo sistêmico dependente do CA, sendo estas: Coarctação da Aorta (COA), interrupção do arco aórtico, síndrome do coração hipoplásico esquerdo, Drenagem Anômala de Veias Pulmonares (DAVP), dupla via de saída do ventrículo direito, coração univentricular e Truncus Arteriosus. Elas representam 
maior número de casos, comprometendo o sistema hemodinâmico em longo prazo (Aragão et al., 2013; Silva, Aguiar, Cunha \& Rodrigues, 2014; Belo, Oselame \& Neves, 2016; Saxena et al., 2019).

Cerca de 1-2\% dos neonatos com CC são considerados críticos, e destes cerca de 30\% morrem sem o diagnóstico precoce, devido ao fechamento fisiológico do CA que ocorre geralmente nas primeiras horas de vida, justificado pelas reações químicas, desencadeadas no organismo do feto um pouco antes do, durante e imediatamente após o parto (Pedra et al., 2019; Saxena et al., 2019).

Entre os casos mais graves, cabe mencionar a DATVP, em que é necessária a correção cirúrgica para conectar as veias pulmonares que transportam o sangue oxigenado para o átrio esquerdo. Na anomalia da válvula tricúspide de Ebstein, é necessária a reparação e/ou plástica valvar e reparo de ventrículo esquerdo. $\mathrm{Na} \mathrm{TF}$, ocorre o defeito em quatro estruturas anatômicas do coração: estenose da valva pulmonar, hipertrofia do ventrículo direito, Comunicação Interventricular (CIV) e uma extraposição da aorta. Esses são defeitos graves, que necessitam de criteriosa assistência e avaliação multidisciplinar emergencial a depender dos defeitos anatômicos, podendo ainda necessitar de intervenções hemodinâmicas emergenciais como no caso da DATVP, em que é imprescindível que haja uma Comunicação Interatrial (CIA) para a sobrevivência do neonato (Pedra et al., 2019; Saxena et al., 2019).

Destarte, alguns procedimentos podem ser realizados pelo serviço de hemodinâmica em caráter emergencial, como: abertura do septo atrial e dilatação das estenoses por meio de cateter balão que são muitas vezes medidas paliativas, coadjuvantes para o prognóstico dos neonatos até que esses sejam submetidos a correção cirúrgica efetiva (Pedra et al., 2019; Saxena et al., 2019). O desenvolvimento/crescimento do neonato com CC em sua maioria pode apresentar alterações morfofuncionais, uma vez que se trata de um corpo em que o coração anormal pode ocasionar nos sistemas corpóreos algum déficit estrutural ou funcional. O desenvolvimento, pode ser prejudicado ainda pela absorção de nutrientes comprometida, corroborando com diferentes níveis de comprometimento estrutural e/ou funcional dos demais órgãos e sistemas corpóreos (Khokhar, Gowa, Bangash \& Tahir, 2019).

Outra circunstância relatada por Urakawa e Kobayashi (2012), que geralmente se vincula às CCs envolve o baixo peso ao nascer (neonatos com $\leq 2.500$ gramas). As variações de peso entre os neonatos podem ocorrer devido às diferentes circunstâncias gestacionais, como ambiente intrauterino, estado nutricional da gestante, presença de comorbidades maternas, entre outras, sendo, portanto, o peso considerado um reflexo de saúde desejável ou não ao RN.

Em um contexto geral, as causas do surgimento das CCs envolvem heranças multifatoriais, fatores genéticos ou ambientais. Entretanto, sabe-se que, em sua maioria, elas não possuem uma causa conhecida ou preexistente, e os fatores de riscos não são evidentes (Melo et al., 2012). O enfermeiro atuante na UTI-N tem a capacidade e o respaldo legal através da Lei n. 7.498, de 25 de junho de 1986, para atuar de forma mais incisiva e com autonomia, desde que habilitado para exercer e praticar o cuidado em alta complexidade, além de gerir e coordenar as ações e cuidados da equipe técnica (Brasil, 1986, 2017c).

$\mathrm{Se}$, durante o exame físico, avaliação clínica e/ou prestação de cuidados ao RN, o enfermeiro suspeitar que o neonato possa ser portador de alguma CC, este deverá traçar imediatamente um plano de cuidados assistenciais capaz de abarcar as peculiaridades e demandas relacionadas às CCs, mesmo antes da confirmação médico-diagnóstica. Isso se justifica pela necessidade de resguardar o RN até que haja a confirmação diagnóstica, minimizando-se possíveis danos à saúde da criança (Brasil, 2017b; Pedra et al., 2019).

Algumas das condições clínicas que podem contribuir com a ocorrência de CC que acarretam riscos aos neonatos são: diabetes gestacional; fenilcetonúria materna; ingestão materna de medicamentos - Inibidor da Enzima de Conversão de Angiotensina (ECA), ácido retinoico, Anti-Inflamatórios Não Esteroides (Aines); rubéola materna; histórico familiar; uso de 
drogas (i)lícitas materna; miocardite gestacional; cariótipo fetal anormal e Batimento Cardiofetal (BCF) anormal (Rocha et al., 2018; Bell et al., 2019; Pedra et al., 2019).

O diagnóstico deve ocorrer ainda durante a triagem fetal por meio do exame de Ultrassonografia (USG) obstétrica e morfológica, sendo estas realizadas em dois momentos: no $1^{\circ}$ trimestre (entre 11 e 14 semanas de gestação) e no $2^{\circ}$ trimestre (entre 20 e 24 semanas de gestação) como integrantes da rotina de pré-natal, conforme recomendação do Sistema Único de Saúde (SUS). Outro exame é a ecocardiografia fetal, porém ainda não disponível na rede SUS. É importante ressaltar que, para o rastreio das CCs, a melhor ferramenta, na atualidade, é a ecocardiografia fetal, realizada no período pré-natal, por volta da $28^{\text {a }}$ semana de gestação. Contudo, esse exame ainda não está disponível no SUS, devido ao seu alto custo tendo em vista a utilização de tecnologias especializadas e demanda por médicos especialistas e capacitados para sua execução (Brasil 2017b, Pedra et al., 2019).

Esses exames possibilitam a visualização das quatro câmaras cardíacas, dos grandes vasos e do arco aórtico; fato esse que favorece o rastreio precoce intrauterino das CCs (Brasil, 2017a, b). Nesse contexto, cabe mencionar que os procedimentos de USG obstétricos, a partir da Resolução do Conselho Federal de Enfermagem (Cofen) $n^{\circ}$ 627/2020, também são procedimentos que podem ser realizados por enfermeiros obstetras capacitados, o que representou um ganho e um avanço para a enfermagem (Brasil, 2020b).

Cabe mencionar ainda que toda gestante cujo feto apresente alterações morfológicas cardiovasculares detectadas durante a triagem neonatal deverá ser referenciada aos centros especializados para o adequado acompanhamento. Destaca-se ainda a interferência de vários fatores que podem dificultar o diagnóstico dessas alterações pela ultrassonografia morfológica, como: expertise profissional; Idade Gestacional (IG); posição fetal; tipo de CC, etc. Ressalta-se ainda a necessidade da detecção precoce das CCs com estratificação de sua gravidade, para que se possa planejar o processo de parto e/ou, em casos mais graves, a realização da cirurgia ainda que na fase intrauterina (Brasil, 2017b; Pedra et al., 2019).

Um importante exame de triagem neonatal é o teste do coraçãozinho ou oximetria de pulso, realizado pelo enfermeiro e/ou médico, que objetiva verificar a saturação arterial de oxigênio, sendo um método que facilita a monitorização contínua, por meio de uma prática não invasiva, capaz de detectar as CCs. O exame é realizado nos neonatos com IG $\geq 34$ semanas, durante as primeiras 24-48 horas de vida, com a finalidade de averiguar as saturações, pré e pós-ductais nos RNs (Plana et al., 2018; Brasil, 2017b; Pedra et al., 2019). Evidências (inter)nacionais apontam que com esses exames se atingiu um efetivo alcance diagnóstico, favorecendo mais segurança no parto e diminuição dos riscos de mortalidade materno-infantil (Pedra et al., 2019, Saxena et al., 2019).

Os sinais clínicos mais comuns para a deteç̧ão em neonatos cardiopatas envolvem: arritmia, cianose, sopro cardíaco, baixo débito cardíaco, taquipneia progressiva, fadiga durante a amamentação, palidez cutânea, sudorese, taquicardia, redução do fluxo sanguíneo dos pulsos centrais/periféricos e hipotensão arterial (Silva et al., 2014). Esse conjunto de sintomas clínicos deve ser identificado precocemente por quaisquer profissionais da equipe interdisciplinar intensiva, na qual a enfermagem recebe destaque, visto a sua assistência integral à beira leito 24 horas por dia (Brasil, 1986, Pedra et al., 2019).

O tratamento medicamentoso envolve o uso de Prostaglandina E-1 para vasodilatação do CA estenosado; no controle da Pressão Arterial (PA), podem ser prescritos pelo médico: Dopamina, Dobutamina, Epinefrina, Sildenafil e Captopril; na ocorrência de congestão pulmonar, sugere-se o uso de diuréticos, como a Furosemida (Silva et al., 2014; Khalil et al., 2019). A enfermagem possui papel de destaque em relação à terapia farmacológica de modo a assistir/cuidar no que tange aos processos de aprazamento, preparo, administração, checagem, monitorização quanto a possíveis complicações, reações adversas e/ou interações medicamentosas (Brasil, 2012; 2017 a, b, Pedra et al., 2019).

Entre as comorbidades evidenciadas em neonatos com CC que necessitam de uma intervenção cirúrgica, destacam-se miocardites, alterações estruturais das fibras miocárdicas (mucopolissacaridoses ou glicogenoses) secundárias ao diabetes 
gestacional materno e fatores relacionados a condições genéticas (Khalil et al., 2019; Pedra et al., 2019). A correção definitiva das CCs, bem como o controle dos sinais e sintomas com a promoção de melhorias sobre a qualidade de vida, é otimizada mediante o tratamento cirúrgico dessas CCs (Silva, Stipp, Pereira, Paes \& Knupp, 2018). A escolha por uma intervenção cirúrgica em neonatos com CC dependerá da confirmação diagnóstica, da técnica operatória escolhida e do planejamento da assistência perioperatória, o que vai muito além do procedimento cirúrgico propriamente dito (Jesus et al., 2018).

Cabe mencionar ainda que as cirurgias intrauterinas são uma opção eficaz em muitos casos de CC, porém se restringem a um pequeno número possível de casos a se realizar o procedimento, devido à gravidade da doença, complexidade do procedimento e condições clínicas do binômio gestante/feto (Pedra et al., 2019).

Segundo Urakawa e Kobayashi (2012), a maturação de órgãos vitais como pulmões, cérebro e o próprio coração deve ser priorizada no período pós-cirúrgico pós-parto, pois é nesse momento que esses órgãos serão influenciados pela correção da cirurgia e terão suas funcionalidades definidas. Isso resulta em amplos benefícios ao neonato. A conduta cirúrgica deve ser definida e compartilhada entre o médico cirurgião e os familiares/responsáveis pelo neonato. Cabe mencionar que a enfermagem coparticipa de todo o processo de cuidado em todas as etapas do período perioperatório, desde a decisão até o planejamento dos cuidados pós-alta (Jesus et al., 2018, Silva et al., 2018; Gozzola, Leite \& Gonçalves, 2020).

A gravidade de uma CC se dá por suas fragilidades fisiológicas, que são oportunidades para desenvolvimento de outras patologias e/ou juntamente com agravamento das patologias que são preexistentes. Sendo assim, os neonatos com CC têm a probabilidade de apresentar alterações que logo desencadeiam outras ocorrências críticas. Diagnosticar e interferir precoce e interdisciplinarmente são formas de agilizar/otimizar o manejo clínico com melhorias no prognóstico do neonato com CC (Pedra et al., 2019).

Independentemente da conduta cirúrgica imediata, a maioria dos neonatos com CC são encaminhados após o parto para a UTI-N devido à necessidade de receber suporte específico, monitorização contínua e cuidados especializados adequados. Nessa fase inicial, um fator que leva o neonato para a UTI é a prematuridade, que está associada à imaturidade dos órgãos vitais, acarretando déficits na oxigenação sistêmica e principalmente no pulmão e coração, circunstâncias estas que requerem tratamento intensivo imediato (Martin, Cross, Hom \& Klugman, 2019; Mebius et al., 2020).

A atuação da enfermagem se inicia na entrada da/o gestante/neonato no ambiente hospitalar, após o nascimento ou internação do mesmo no contexto intensivo, quando são empregados as intervenções e os cuidados da equipe multiprofissional. Urakawa e Kobayashi (2012) ressaltam que o cuidado de enfermagem ocorre dentro do Processo de Enfermagem (PE) e de forma estruturada nas etapas da Sistematização da Assistência de Enfermagem (SAE). Esta objetiva uma assistência segura, de qualidade e com fundamentação teórica, científica e metodológica, capaz de garantir um cuidado numa visão holística, assegurando um planejamento do cuidado de enfermagem eficaz na redução de agravos à saúde e mortalidade neonatal (Oster et al., 2016).

A execução da SAE como parte integrante do PE proporciona uma organização do ambiente de trabalho na qual a equipe de enfermagem exerce suas atividades, organizando as funções e cada membro da equipe com uma divisão e estabelecimento de linhas de cuidados com a estratificação de prioridades e metas a serem atingidas. Assim é assegurado à pessoa cuidada, a seus familiares e à equipe de saúde respaldo legal para o exercício legal da profissão (Brasil, 1986, 2009; Oster et al., 2016; Melo et al., 2021).

A enfermagem é fundamental no atendimento intensivo ao neonato, mediante a identificação de demandas por cuidados críticos. Para isso, faz-se necessário à equipe um arcabouço de conhecimentos teóricos e científicos, preparo técnico/prático, sensibilidade, atenção nas questões multidimensionais num conceito ampliado de saúde, como exemplo o cuidado psicológico da díade familiares/criança para que possa intervir de forma rápida e eficaz (Urakawa \& Kobayashi, 2012). 
Pode-se perceber que os cuidados de enfermagem intensivos a neonatos com CC devem ser sistematizados e precisos; logo, o enfermeiro possui papel fundamental para que o manejo clínico da criança com CC seja seguro e de qualidade, promovendo um suporte terapêutico elementar na evolução do quadro clínico, hemodinâmico, bem como reabilitação intensiva e prognóstico de saúde, visando à qualidade de vida e ao conforto necessário à díade neonato/familiar (Hickey et al., 2016).

Destarte, é imprescindível que se considere a família como parte integrante do cuidado em busca de informações e que esta seja incluída no planejamento da assistência, bem como que sejam fornecidas todas as orientações e esclarecidas as dúvidas apresentadas pelos familiares a respeito do tratamento (Krempser, Caldas, Arreguy-Sena, Melo \& Krepker, 2020a; Krempser, Caldas, Arreguy-Sena \& Melo, 2020b).

Os principais diagnósticos de enfermagem possíveis que abrangem os neonatos com CC evidenciados são: risco de infecção; troca de gases prejudicada e intolerância à atividade (Herdman \& Katmitsuru, 2018). Os RNs com CC são mais suscetíveis a infecções devido a sua baixa capacidade imunológica correlacionada ao tempo de internação intensiva, desnutrição, uso de medicamentos à base de corticoides e quantitativo de procedimentos invasivos vivenciados. A troca de gases prejudicada tem sua associação à imaturidade pulmonar e à insuficiência cardiopulmonar. Já a intolerância à atividade envolve os esforços (sucção do leite materno durante a mamada; ao chorar, ao realizar as necessidades fisiológicas, como na micção e evacuação e nos movimentos corpóreos), os quais podem gerar fadiga neonatal (Silva, Pereira, Figueiredo, Guimarães \& Cavalcanti, 2015).

O fato de o enfermeiro conhecer as características do neonato com CC favorece a prestação da assistência de enfermagem direcionada pelos diagnósticos de enfermagem (Brasil, 2009; Silva et al., 2015). Desse modo, entre as intervenções e os cuidados de enfermagem, cabe destaque para monitorização clínica e hemodinâmica contínua, principalmente a oximetria de pulso nos primeiros momentos de vida; anamnese cardiovascular qualificada; avaliação da perfusão sanguínea central e periférica; promoção de assistência perioperatória adequada ao tipo de cirurgia, quadro clínico e etapa cirúrgica; cuidados em relação à manutenção da temperatura, incubadora, higiene corporal e conforto; oxigenoterapia; administração da amamentação conforme prescrição; administração da terapia farmacológica; realização de procedimentos exclusivos do enfermeiro, como a viabilização de Cateter Intravascular (CIV), passagem de sondas e cateteres, etc.; assistência às intercorrências com registros das mesmas, entre outros (Melo et al., 2012).

Sendo assim, a enfermagem como parte integrante e constituinte da equipe multiprofissional e interdisciplinar atua com vistas à diminuição/eliminação da possibilidade de complicações e/ou sequelas e traumas decorrentes do cuidado intensivo. Cabe destacar ainda que o acolhimento deve ser humanizado, com escuta qualificada nas relações com os familiares/pais do neonato e possuir um caráter orientador, de aconselhamento, sendo fonte de apoio, preparando os mesmos para os processos de aceitação e enfrentamento da condição clínica do neonato. A família do neonato deve ser integrada se possível nas ações de cuidado, otimizando-se as condições favoráveis para que a criança possa crescer e se desenvolver com qualidade de vida e saúde (Melo et al., 2012; Magalhães, Chaves \& Queiroz, 2019).

\section{Considerações Finais}

Ao refletir sobre os principais cuidados intensivos de enfermagem cardiovascular a neonatos portadores de CC, conclui-se que a atuação do enfermeiro e sua equipe na abordagem das CCs no contexto intensivo é sistematizada no PE e deve ocorrer integrada à atuação dos demais profissionais e sempre de forma interdisciplinar, visando a uma assistência segura, de qualidade, eficaz e humanizada.

Os principais cuidados críticos de enfermagem abarcaram a atuação na prevenção de doenças, promoção da saúde, diagnóstico precoce de forma clínica, laboratorial e por imagem, bem como nos processos de tratamento clínico, farmacológico 
ou cirúrgico. Estes se dão em prol da reabilitação, cura ou do estabelecimento de um prognóstico favorável à criança, visando também ao cuidado dos familiares da criança por meio de uma atuação sempre integrada à equipe interdisciplinar.

Foi considerada como provável limitação desta investigação a impossibilidade de realizar uma pesquisa de campo visto o momento pandêmico atual de enfrentamento global da covid-19. Para reduzir essa limitação, a estratégia adotada foi a de uso de um maior número de descritores como estratégia de busca, bem como a adoção de um recorte de tempo de dez anos para a construção de um consolidado de evidências na elaboração da síntese do conhecimento científico capaz de exprimir o estado da arte em resposta ao objetivo desta investigação.

Ressalta-se ainda a escassez de produção científica da enfermagem sobre o objeto investigado, indexada nas bases de dados (inter)nacionais. Diante disso, evidenciou-se a necessidade de realização de novas investigações no campo da enfermagem cardiovascular, neonatal e intensiva em diferentes realidades e delineamentos metodológicos capazes de contribuir com o estado da arte a respeito da assistência de enfermagem a neonatos, de modo a retratarem diferentes estratégias de cuidados cardiovasculares na abordagem intensiva das CCs sendo estas lacunas do conhecimento científico identificadas as recomendações dos autores para a realização de novos trabalhos futuros sobre a temática.

Acredita-se que esta investigação possa contribuir para o campo da enfermagem e saúde, mediante apresentação da síntese do conhecimento científico, que oportuniza reflexões sobre os principais cuidados intensivos que o enfermeiro e sua equipe devem realizar ao neonato com CC de modo a prevenir/amenizar possíveis agravamentos clínicos e promover um melhor prognóstico de cura e/ou reabilitação segura, eficaz e de qualidade.

\section{Referências}

Anwar, S., Singh, G. K., Miller, J., Sharma, M., Manning, P., Billadello, J. J., \& Woodard, P. K. (2018). Impressão 3D é uma tecnologia transformadora em doenças cardíacas congênitas. Jacc Basic Transl Sci., 3 (2), 294-312.

Aragão, J. A., Mendonça, M. P., Silva, M. S., Moreira, A. N., Aragão, M. E. C. S., \& Reis, F. P. (2013). O Perfil Epidemiológico dos Pacientes com Cardiopatias Congênitas Submetidos à Cirurgia no Hospital do Coração. Revista Brasileira de Ciências da Saúde, 17 (3), $263-8$.

Araújo Neto, J. D. A., Silva, I. S. P., Zanin, L. E., Andrade, A. P., \& Moraes, K. M. (2016). Profissionais de saúde da unidade de terapia intensiva percepção dos fatores restritivos da atuação multiprofissional. Revista Brasileira em Promoção da Saúde, 29 (1), 43-50.

Bell, J. L., Saenz, L., Domnina, Y., Baust, T., Panigrahy, A., Bell, M. J., \& Sanches-deToledo, J. (2019). Acute Neurologic Injury in Children Admitted to the Cardiac Intensive Care Unit. Ann Thorac Surg, (1) 107, 1831-7.

Brasil. (2020a). Resolução $C F M n^{\circ}$ 2.271/2020: define as unidades de terapia intensiva e unidades de cuidado intermediário conforme sua complexidade e nível de cuidado, determinando a responsabilidade técnica médica, as responsabilidades éticas, habilitações e atribuições da equipe médica necessária para seu adequado funcionamento. Conselho Federal de Medicina (CFM).

Brasil. (2020b). Resolução COFEN $n^{\circ}$ 627/2020: normatiza a realização de Ultrassonografia Obstétrica pelo Enfermeiro Obstétrico. Conselho Federal de Enfermagem (COFEN).

Brasil. (2018). Agenda de prioridades de pesquisa do Ministério da Saúde - APPMS. - Brasília: DF. Ministério da Saúde (MS). Secretaria de Ciência, Tecnologia e Insumos Estratégicos. Departamento de Ciência e Tecnologia.

Brasil. (2017a). Síntese de evidências para políticas em saúde: diagnóstico precoce de cardiopatias congênitas. Brasília: MS- EVIPNet Brasil. - Ministério da Saúde (MS): Secretaria de Ciência, Tecnologia e Insumos Estratégicos. Departamento de Ciência e Tecnologia.

Brasil. (2017b). Portaria n ${ }^{\circ}$ 1.727/2017: aprova o Plano Nacional de Assistência à Criança com Cardiopatia Congênita. - Ministério da Saúde (MS): Secretaria de Atenção à saúde.

Brasil. (2017c). Resolução COFEN n 564/2017: dispõe sobre o novo Código de Ética dos Profissionais de Enfermagem. - Conselho Federal de Enfermagem (COFEN).

Brasil. (2012). Atenção à Saúde do Recém-Nascido. Ministério da Saúde (MS). Secretaria de Atenção à saúde. Departamento de Ações Programáticas Estratégicas. 
Brasil. (2010). RDC $N^{o} 7$ de 24 de fevereiro de 2010: dispõe sobre os requisitos mínimos para funcionamento de Unidades de Terapia Intensiva e dá outras providências. - Agência Nacional de Vigilância Sanitária (ANVISA).

Brasil. (2009). Lei 358/2009: dispõe sobre a Sistematização da Assistência de Enfermagem e a implementação do Processo de Enfermagem. - Conselho Federal de Enfermagem (COFEN).

Brasil. (1986). Conselho Federal de Enfermagem (COFEN). Lei 7498/86: dispõe sobre a regulamentação do exercício da enfermagem e dá outras providências. - COFEN.

Belo, W. A., Oselame, G. B., \& Neves, E. B. (2016). Perfil clínico-hospitalar de crianças com cardiopatia congênita. Cad. Saúd Colet, 24(2):216-20.

Gozzola, L. P. L., Leite, H. V., \& Gonçalves, G. M. (2020). Comunicando más notícias sobre malformações congênitas: reflexões bioéticas e jurídicas. Rev. Bioét., 28 (1), 38-46.

Herdman, T. H., \& Katmitsuru, S. Diagnósticos de enfermagem da NANDA: definições e classificação 2018 a 2020. Artmed, 2018-2020.

Hickey, P. A., Pasquali, S. K., Gaynor, J. W., Jacobs, M. L., Jacobs, J. P., \& Hirsch-Romano, J. C. (2016). Impacto da enfermagem em cuidados intensivos nos resultados de pacientes pediátricos. Ann Thorac Surg, 102 (4), 1375-80.

Hockenberry, M. J., Wilson, D. W., \& Rodgers, C. C. Wong-Fundamentos de Enfermagem Pediátrica. (10a ed.), Elsevier, 2018.

Jesus, V. S. D., Nascimento, A. M., Miranda, R. D. A., Lima, J. S., Tyll, M. A. G., \& Veríssimo, A. O. L. (2018). Fila de Espera para Tratamento de Pacientes com Cardiopatia Congênita: Retrato de um Centro de Referência Amazônico. Int J Cardiovasc Sci., 31 (4), 374-82.

Khalil, M., Jux, C., Rueblinger, L., Behrje, J., Esmaeili, A., \& Scharanz, D. (2019). Terapia aguda de recém-nascidos com doença cardíaca congênita crítica. Translational Pediatrics, 8 (2), 114-26.

Khokhar, R. A., Gowa, M. A., Bangash, S. K., \& Tahir, A. (2019). O espectro de procedimentos cardíacos pediátricos e seus resultados: um relatório de seis meses da maior instalação cardíaca. Cureus, 11 (8), e5339.

Krempser, P., Caldas, C. P., Arreguy-Sena, C., Melo, L. D., \& Krepker, F. F. (2020a). Maintenance of peripheral venipuncture in children: perspectives of nursing professionals and companions. Research, Society and Development, 9(11), e849119600-e849119600.

Krempser, P., Caldas, C. P., Arreguy-Sena, C., \& Melo, L. D. (2020b). Social representations and pediatric venus puncture stressors: contributions to nursing care. Enfermagem em Foco, 11 (4), 15-21.

Magalhães, S. S., Chaves, E. M. C., \& Queiroz, M. V. O. (2019). Design instrucional para o cuidado de enfermagem aos neonatos com cardiopatias congênitas. Texto contexto - enferm. 1 (28), e20180054.

Marques, T. O., Melo, L. D., Taroco, F. E., Duarte, R. M. L., \& Lima, H. D. (2021). Serviços de Atendimento Móvel de Urgência (Samu): uma revisão integrativa. Research, Society and Development, 10 (2), e38310212522-e38310212522.

Martin, G. R., Cross, R. R., Hom, L. A., \& Klugman, D. (2019). Updates in Congenital Heart Disease: Can Outcomes Improve? Am J Perinatol., 36 (02), $22-$ S28.

Mebius, M. J., Bilardo, C. M., Kneyber, M. C. J., Modestini, M., Ebels, T., Berger R. M. F., \& Kooi, E. M. W. (2020). Início de lesão cerebral em bebês com cardiopatia congênita diagnosticada no pré-natal. PLoS One, 15 (3), e0230414.

Melo, L. D., Silva, D. A., \& Jeremias, J. S. (2021). Patient systematic intensive care in post-operative cardiac. J. Res.: Fundam. Care. Online, 1(13), 467-76.

Melo, L. D., Carvalho, A. W., Mello, J. I. B. V., Santos, P. R., Mozzer, D. D., \& Taroco, F. E. (2020). Intensive care to people with heart failure decompensed in the prevention and treatment of acute pulmonary edema. Revista Estação Científica, 24:1-20.

Melo, H. C., Araújo, S. E. G., Santos V. E. F. A., Veríssimo, A. V. R., Alves, E. R. P., \& Souza, M. H. N. (2012). Ser-Enfermeiro em face do cuidado à criança no pós-operatório imediato em cirurgia cardíaca: pesquisa qualitativa. Esc Anna Nery, 16 (3), 473-9.

Oster, M. E., Aucott, S. W., Glidewell, J., Hackell, J., Kochilas, L., Martin, G. R., \& Kemper, A. R. (2016). Lições aprendidas com a triagem neonatal para cardiopatias congênitas críticas. Pediatrics. 137 (5), e20154573.

Papalaia, D. E., \& Feldman, R. D. Desenvolvimento humano. (12a ed.), Amgh.

Pedra, S. R. F. F., Zielinsky, P., Binotto, C. N., Zielinsky, P., Binotto, C. N., Martins, C. N., \& Zamith, M. M. (2019). Diretriz Brasileira de Cardiologia Fetal 2019. Arq Bras Cardiol. 112(5):600-648.

Pinto Junior, V. C. P., Branco, K. M. P. C., Cavalcante, R. C., Carvalho Junior, W., Lima, J. R. C., Freitas, S. M., \& Souza, N. M. G. (2015). Epidemiologia da cardiopatia congênita no Brasil. Rev Bras Cir Cardiovasc, 30 (2), 219-24. 
Research, Society and Development, v. 10, n. 5, e52310515346, 2021

(CC BY 4.0) | ISSN 2525-3409 | DOI: http://dx.doi.org/10.33448/rsd-v10i5.15346

Plana, M. N., Zamora J., Suresh., Fernandez-Pineda, L., Thangaratinam, S., \& Ewer, A. K. (2018). Rastreamento de oximetria de pulso para defeitos cardíacos congênitos críticos. Cochrane Database Syst Rev., 3 (3), 1-84.

Rocha, L. A., Froio, S. C., Silva, C. C., Figueira, S. A. N., Guilhen, J. C. S., Guinsburg, R., \& Araújo Júnior, E. (2018). Fatores de risco para mortalidade em crianças com cardiopatia congênita atendidas em um centro terciário brasileiro. Braz J Cardiovasc Surg. 33 (6), 603-7.

Saxena, A., Relan, J., Agarwal, R., Awasthy, N., Azad, S., Chakrabarty, M., \& Vijaykumar, R. (2019). Indian guidelines for indications and timing of intervention for common congenital heart diseases: Revised and updated consensus statement of the Working group on management of congenital heart diseases. Ann Pediatr Cardiol, 12(3):254-86.

Silva, A. C. S. S., Stipp, M. A. C., Pereira. F. M. V., Paes, G. O., \& Knupp, V. M. A. O. (2018). Variáveis clínicas e laboratoriais associadas ao desfecho mortalidade no pós-operatório de cirurgia cardíaca pediátrica. Esc. Anna Nery, 23 (1), e20180147.

Silva, V. G., Pereira, J. M., Figueiredo, L. S., Guimarães, T. C. F., \& Cavalcanti, A. C. D. (2015). Diagnósticos de Enfermagem em crianças com cardiopatias congênitas: mapeamento cruzado. Acta Paul Enferm, 28 (6), 524-30.

Silva, M. G. P., Aguiar, L. R. S., Cunha, K. J. B., \& Rodrigues, T. K. A. (2014). Caracterização do diagnóstico e tratamento farmacológico das cardiopatias congênita neonatal: acianogênica e cianogênica. $R$. Interd. 7 (4), 146-56.

Sousa, L. M. M., Firmino, C. F., Marques-Vieira, C. M. A., Severino, S. S. P., \& Pestana, H. C. F. C. (2018). Revisões da literatura científica: tipos, métodos e aplicações em enfermagem. Revista Portuguesa de Enfermagem de Reabilitação, 1(1):45-54.

Tessmer, C. S., Rutz, P. A., Bartschi, G. R. I., Alves, B. C., Portella, R. J., \& Soares, M. M. (2020). Types of literature review: considerations of the editors of the Journal of Nursing and Health. J. Nurs. Health. 10, e20104031.

Urakawa, I. T., \& Kobayashi, R. M. (2012). Identificação do perfil e diagnósticos de enfermagem do neonato com cardiopatia congênita. R. Pesq.: Cuid. Fundam. Online, 4 (4), 3118-24. 